\title{
KULEUVEN
}

\begin{tabular}{|c|c|}
\hline Citation & $\begin{array}{l}\text { F. De Groote, G. Pipeleers, I. Jonkers, B. Demeulenaere, C. Patten, J. } \\
\text { Swevers, and J. De Schutter, (2009), } \\
\text { A physiology based inverse dynamic analysis of human gait: potential } \\
\text { and perspectives } \\
\text { Computer Methods in Biomechanics and Biomedical Engineering, 12(5), 563- } \\
574 .\end{array}$ \\
\hline Archived version & $\begin{array}{l}\text { Author manuscript: the content is identical to the content of the published } \\
\text { paper, but without the final typesetting by the publisher }\end{array}$ \\
\hline Published version & http://dx.doi.org/10.1080/10255840902788587 \\
\hline Journal homepage & http://www.tandfonline.com/toc/gcmb20/current \\
\hline Author contact & $\begin{array}{l}\text { friedl.degroote@kuleuven.be } \\
+32(0) 16372682\end{array}$ \\
\hline IR & https://lirias.kuleuven.be/handle/123456789/211761 \\
\hline
\end{tabular}

(article begins on next page) 


\title{
RESEARCH ARTICLE
}

\section{A physiology based inverse dynamic analysis of human gait: potential and perspectives.}

\author{
F. De Groote ${ }^{a}{ }^{*}$, G. Pipeleers ${ }^{a}$, I. Jonkers ${ }^{b}$, B. Demeulenaere ${ }^{a}$, C. Patten ${ }^{c}$, J. Swevers $^{a}$, \\ J. De Schutter ${ }^{a}$ \\ ${ }^{a}$ Dept. of Mechanical Engineering, Katholieke Universiteit Leuven, Leuven, Belgium; \\ ${ }^{b}$ Dept. of Kinesiology, Katholieke Universiteit Leuven, Leuven, Belgium; ${ }^{c}$ Brain \\ Rehabilitation Research Center, Malcom Randall VA Medical Center, USA; Departments \\ of Physical Therapy, Applied Physiology and Kinesiology, and Neurology, University of \\ Florida, USA;
}

(Received July 2008)

\begin{abstract}
One approach to compute the musculotendon forces that underlie human motion is to combine an inverse dynamic analysis with a static optimisation procedure. Although computationally efficient, this classical inverse approach fails to incorporate constraints imposed by muscle physiology.

The present paper reports on a physiological inverse approach that combines an inverse dynamic analysis with a dynamic optimisation procedure. This allows the incorporation of a full description of muscle activation and contraction dynamics, without loss of computational efficiency.

A comparison of muscle excitations and MT-forces predicted by the classical and the physiological inverse approach is presented for normal and pathological gait. Inclusion of muscle physiology primarily affects the rate of active muscle force build-up and decay and allows the estimation of passive muscle force. Consequently, it influences the onset and cessation of the predicted muscle excitations as well as the level of co-contraction.
\end{abstract}

Keywords: biomechanics; motion analysis; inverse dynamics; gait

\section{Introduction}

The calculation of the musculotendon (MT-) forces during gross motor function such as gait is complicated by the overactuation of the musculoskeletal system. Consequently, MT-force calculation is often reformulated as an optimisation problem combined with either an inverse or a forward dynamic analysis.

During an inverse dynamic simulation (Crowninshield and Brand 1981; Yamaguchi et al. 1995) the measured movement kinematics and external (ground reaction) forces acting on the musculoskeletal system are prescribed, and the resulting joint reaction forces and moments are calculated. Subsequently, individual muscle forces are computed based on a static optimisation procedure that minimizes an instantaneous performance criterion, e.g., the sum of muscle stress or activation squared across all muscles (Crowninshield and Brand 1981; Anderson and Pandy 2001). This optimisation procedure is called static since the MT-forces at the time

*Corresponding author. Email: friedl.degroote@mech.kuleuven.be

ISSN: 1741-5977 print/ISSN 1741-5985 online

(C) 2008 Taylor \& Francis

DOI: $10.1080 / 1741597 \mathrm{Y} Y \mathrm{xxxxxxxx}$

http://www.informaworld.com 
instant under consideration are calculated independently of other time instants. Its main advantage is computational efficiency.

During a forward dynamic simulation (Hatze 1976, 1981; Yamaguchi and Zajac 1990; Anderson and Pandy 2001) joint moments, muscle forces or excitations are prescribed, and the resulting motion is calculated. The optimisation procedure involves a trajectory tracking algorithm and/or the minimisation of a performance criterion related to the entire motion cycle, e.g., metabolic energy usage. This optimisation procedure is called dynamic since the entire time course of the MTforces is optimized at once, increasing substantially the computational effort.

Due to the static nature of the optimisation procedure, classical inverse approaches (CIA) fail to account for two basic neurophysiological mechanisms influencing the relationship between muscle excitation and the course of MT-force production (Yamaguchi et al. 1995): (1) The muscle activation dynamics (Lieber 1992) describes the nonlinear relation between muscle excitation and muscle activation due to non-homogeneous muscle fiber activation and the dynamic effects in the muscle activation response to changes in excitation. (2) The muscle contraction dynamics (Zajac 1989) describes the nonlinear relation between muscle activation and MT-force, taking into account the nonlinear length and velocity dependency of the force production of the MT-complex. In contrast to the static optimisation procedure of the CIA, the dynamic optimisation procedure of forward approaches allows enforcing muscle activation and contraction dynamics.

A physiological inverse approach (PIA) was proposed (Pipeleers et al. 2008; De Groote et al. 2006) that supplements a CIA with muscle activation and contraction dynamics. The inclusion of muscle physiology links the instantaneous (static) optimisation problems into one large-scale (dynamic) problem. The number of optimisation variables trebles, since muscle excitations and activations need to be included along with the MT-forces. In addition, the inclusion of muscle physiology imposes nonlinear constraints to the optimisation problem. Nonlinear problems are generally considered hard to solve, mainly because of the potential stall of the solver in a local minimum resulting in a high sensitivity of the obtained result to the starting point of the optimisation process. The global optimum of the problem however can be reliably and efficiently found for convex optimisation problems, a subclass of nonlinear optimisation problems. Mathematically, these optimisation problems are characterized by (i) linear equality constraint functions, (ii) convex inequality constraint functions, and (iii) a convex goal function (Boyd and Vandenberghe 2004). Muscle physiology violates the first requirement by imposing nonlinear equality constraints, therefore making the optimisation problem nonconvex. The resulting large-scale nonconvex optimisation problem is solved using initializing for local optimisation. This is a two-step approach: first a hot-start is determined based on solving an approximate convex quadratic optimisation problem, second the nonconvex optimisation problem is solved from the hot start (Pipeleers et al. 2008).

The contribution of the present paper is twofold. First, we introduce a nonlinear change of variables that turns the original large-scale nonconvex optimisation problem into an equivalent convex quadratic problem. This nonlinear change of variables is an improvement over the previously proposed initializing for local optimisation, since it is a one-step approach requiring only a convex quadratic optimisation that is guaranteed to yield the global optimum. Additionally, applying the nonlinear change of variables to the activation dynamics models proposed by Zajac (1989) and Raasch et al. (1997), we show that both models are equivalent in terms of muscle activation and MT-forces. Only the corresponding excitation patterns are different.

Second, the effect of including muscle physiology is discussed based on a compar- 


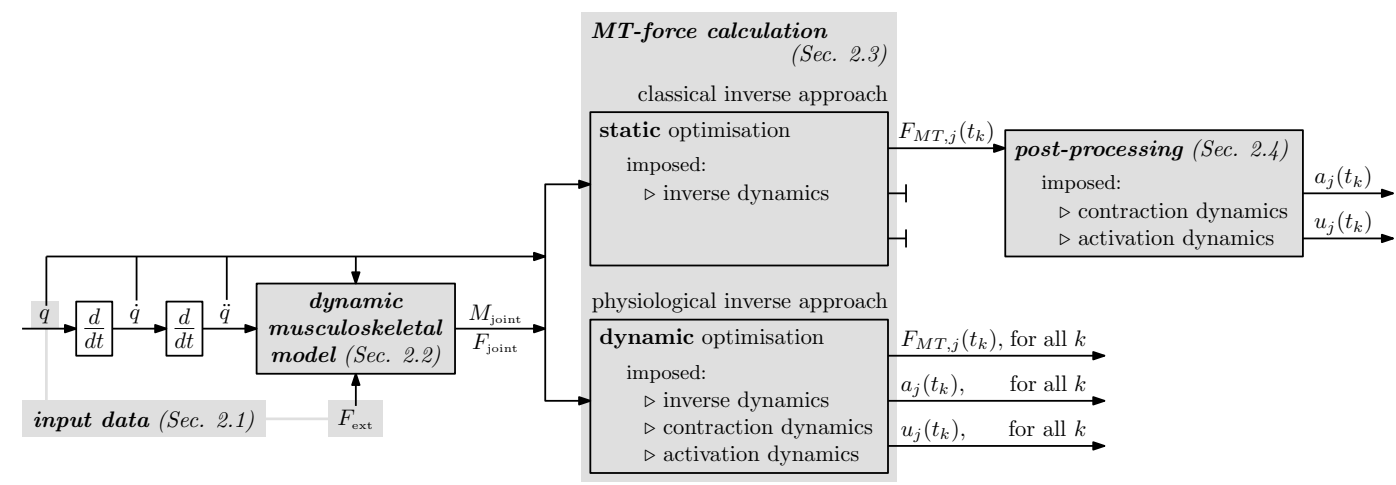

Figure 1. Outline of the physiological and the clasical inverse approach. Both approaches include an inverse dynamic simulation to compute the joint reaction moments and forces $\left(M_{\text {joint }}, F_{\text {joint }}\right)$ from the measured skeleton motion $(q, \dot{q}, \ddot{q})$ and ground reaction forces $\left(F_{\text {ext }}\right)$, using a dynamic musculoskeletal model. The classical inverse approach continues with a static optimisation procedure to calculate MT-forces $\left(F_{M T, j}\right)$ at each separate time instant $t_{k}$. The indices $k$ and $j$ number respectively the time instants included in the simulation and the muscles included in the musculoskeletal model. The physiological inverse approach uses a dynamic optimisation approach to solve for the MT-forces, activations $\left(a_{j}\right)$, and excitations $\left(u_{j}\right)$ at all considered time instants at once.

ison of the muscle excitations and MT-forces computed by the CIA and the PIA for normal and pathological gait. This comparison requires a new post-processing step for the CIA which computes the muscle excitations corresponding to the obtained muscle activations.

\section{Methodology}

For a gait cycle of a control subject $(\mathrm{C})$ and a hemiparetic subject $(\mathrm{H})$, muscle excitations and MT-forces of the major lower limb muscles are calculated for one leg by the PIA and the CIA. Both approaches are outlined in Figure 1.

\subsection{Experimental setup and input data}

Instrumented gait analysis was performed on the control subject and the hemiparetic subject using a 7 camera motion capture system (Qualysis, Inc., Goteborg, Sweden) with 3 synchronized force plates (AMTI, Watertown, MA, USA; and Bertec, Columbus, OH, USA). A modified Cleveland Clinic marker placement protocol was used (38 markers, Figure 2). Simultaneously, the surface EMG of 8 muscle groups was collected: M. biceps femoris, M. rectus femoris, M. vastus lateralis, M. semimembranosus, M. gastrocnemius (medial head), M. tibialis anterior, M. soleus, and M. gluteus medius. The raw EMG signal is high-pass filtered, rectified, and normalized with respect to the maximal signal amplitude over the gait cycle. A minimum of three valid trials was collected for each limb. From inspection of the three trials, a representative trial was selected. All procedures were approved by the Stanford University panels on human subjects in research, and all subjects gave informed consent.

Input to the simulation are the 3D ground reaction forces and moments and the kinematics calculated based on the trajectories of retroflective markers using inverse kinematics. A residual elimination algorithm (Thelen and Anderson 2006) is applied to make the kinematics dynamically consistent with the measured ground reaction forces and moments. The gait cycles take $1.08 \mathrm{~s}(\mathrm{C})$ and $1.41 \mathrm{~s}(\mathrm{H})$, respectively. 

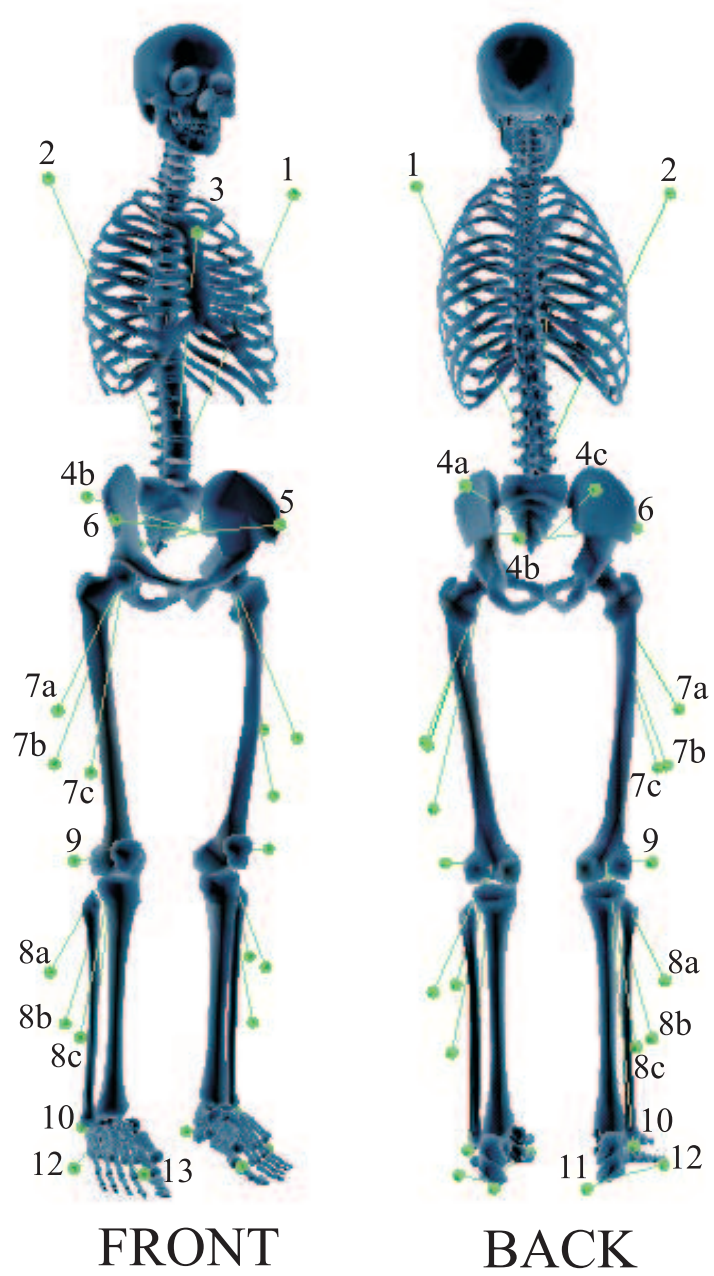

Figure 2. Marker placement protocol. A modified Cleveland marker placement protocol (Sutherland 2002) was used for the data collection. The marker set consisted of 30 markers, including five clusters of three markers. Three anatomical markers defined the trunk: a marker on the lateral aspects of the left (1) and right (2) shoulder and a marker on the sternum (3). The pelvis segment is defined by a cluster of three technical markers on the sacrum $(4 \mathrm{a}, 4 \mathrm{~b}, 4 \mathrm{c})$ and two anatomical markers on the left (5) and right (6) Anterior Superior Iliac Spine (ASIS). The thigh segment is defined by a cluster of three technical markers $(7 \mathrm{a}, 7 \mathrm{~b}, 7 \mathrm{c})$. The shank segment is defined by a cluster of three technical markers $(8 \mathrm{a}, 8 \mathrm{~b}, 8 \mathrm{c})$, an anatomical marker on the lateral epicondyle (9), and an anatomical marker on the lateral malleolus (10). The foot segment is defined by three anatomical markers on the heel (11), the lateral foot (12) and the first metatarsal head (13). During a static calibration trial, additional anatomical markers were added to the medial femoral condyles and the medial malleoli to define the knee and ankle joint axis.

\subsection{Dynamic musculoskeletal model}

The dynamic model consists of eight body segments: a head-arms-trunk (HAT) segment, the pelvis, left and right thigh, lower leg and foot (Delp et al. 1990). The generic model was scaled to the subject's dimensions using marker information collected during a static trial. Segmental mass and inertial parameters are adapted to reflect the test subject's anthropometry (de Leva 1996).

Nineteen degrees of freedom (DOF) are included: A spherical joint connects the HAT-segment to the pelvis and the pelvis to the thighs. The ankle joints are modeled as a simple hinge, whereas the knee joints are modeled as a sliding hinge (Yamaguchi and Zajac 1989). The remaining six DOF correspond to the position and orientation of the pelvis.

Equations of motion are generated in SIMM Dynamics Pipeline. An inverse dynamic analysis by SD Fast (Symbolic Dynamics Inc.) calculates the joint reaction moments and forces. 
Based on the kinematic input, the moment arms of 43 muscle tendon actuators of the lower limb (Delp et al. 1990; Anderson 1999) are calculated at each instant of the recorded movement.

\subsection{MT-Force Calculation}

The CIA as well as the PIA calculate the force contribution $F_{M T, j}$ of each MTactuator $j$. Both methods enforce the MT-force distribution to be consistent with the joint reaction moments using the instantaneous moment arms of the MTactuators.

\subsubsection{Classical Inverse Approach (CIA)}

At each time instant $t_{k}$ a static optimisation problem is solved to calculate the instantaneous force contribution $F_{M T, j}$. The static performance criterion is the sum of the squared activations of all the muscles:

$$
f_{\mathrm{cia}, k}=\sum_{j}\left(a_{j}\left(t_{k}\right)\right)^{2} .
$$

The MT-forces are constrained by:

$$
F_{M T, j}\left(t_{k}\right)=a_{j}\left(t_{k}\right) F_{M T, j}^{\max }\left(t_{k}\right),
$$

where muscle activations $a_{j}$ must lie between 0 and 1 , the values corresponding to respectively minimal and maximal activation, and $F_{M T, j}^{\max }$ denotes the instantaneous maximal force generating capacity of the MT-actuator, calculated from its forcelength-velocity curve and the kinematic input. This implementation is identical to the one used by Anderson and Pandy (2001).

\subsubsection{Physiological Inverse Approach (PIA)}

The PIA relies on a large-scale dynamic optimisation problem, calculating the muscle excitations $u_{j}$, activations $a_{j}$, and MT-forces $F_{M T, j}$ at all the considered time instants simultaneously using direct transcription.

Muscle excitations and activations must lie between 0 and 1 , the values corresponding to respectively minimal and maximal excitation and activation:

$$
\begin{aligned}
& 0 \leq u_{j} \leq 1 \\
& 0 \leq a_{j} \leq 1
\end{aligned}
$$

The MT-forces are enforced to comply with muscle physiology. Two models describing the activation dynamics are retreived from literature:

Zajac (1989):

$$
\frac{\mathrm{d} a_{j}}{\mathrm{~d} t}=\frac{1}{\tau_{a}} u_{j}-\frac{1}{\tau_{d}} a_{j}+\left(\frac{1}{\tau_{d}}-\frac{1}{\tau_{a}}\right) u_{j} a_{j},
$$

and Raasch et al. (1997):

$$
\frac{\mathrm{d} a_{j}}{\mathrm{~d} t}= \begin{cases}\left(u_{j}-a_{j}\right)\left(\frac{u_{j}}{\tau_{a}}+\frac{1-u_{j}}{\tau_{d}}\right) & u_{j} \geq a_{j} \\ \frac{u_{j}-a_{j}}{\tau_{d}} & u_{j}<a_{j}\end{cases}
$$


with activation time constant $\tau_{a}=11 \mathrm{~ms}$ and deactivation time constant $\tau_{d}=68 \mathrm{~ms}$ (Winters and Stark 1988).

The contraction dynamics are imposed by a linearized Hill-model, neglecting muscle fiber contraction speed (Pipeleers et al. 2008). The model of Hill (Zajac 1989) describes the contraction dynamics by a nonlinear first order differential equation in $F_{M T, j}$ :

$$
\frac{d F_{M T, j}}{d t}=f_{\mathrm{dif}, \mathrm{j}}\left(F_{M T, j}, a_{j}, t\right) .
$$

This model is based on five muscle-specific parameters for which the values proposed by Delp et al. (1990) are used in this study. For gait, the influence of the lengthening speed of the muscle fibers on the musculotendon force is considered negligible. As a result, the nonlinear differential equation 7 can be accurately approximated by a nonlinear algebraic equation:

$$
F_{M T, j}\left(t_{k}\right)=f_{\mathrm{alg}, \mathrm{j}}\left(a_{j}\left(t_{k}\right), l_{M T, j}\left(t_{k}\right)\right),
$$

where $l_{M T, j}\left(t_{k}\right)$ is the musculotendon length at time instant $k$, calculated from the measured kinematics and the musculoskeletal model. This equation is only slightly nonlinear and hence, is approximated very well by the linearized model:

$$
F_{M T, j}\left(t_{k}\right)=c_{j, k} a_{j}\left(t_{k}\right)+d_{j, k} .
$$

$d_{j, k}$ and $c_{j, k}$ are time and muscle-dependent constants. $d_{j, k}$ denotes the MT-force predicted by the model of Hill (8) in the continued absence of activation, that is, the instantaneous passive MT-force:

$$
d_{j, k}=f_{\text {alg,j }}\left(0, l_{M T, j}\left(t_{k}\right)\right) .
$$

$c_{j, k}$ minimizes, using a least squares approach, the difference in activations calculated using the linearized (9) and original Hill-model (neglecting muscle fiber contraction speed) (8) for 21 musculotendon forces, equidistantly divided between the passive and maximal active musculotendon force at time instant $t_{k}$.

The dynamic performance criterion sums (1) over all time instants $t_{k}$ :

$$
f_{\text {pia }}=\sum_{k} f_{\text {cia }, k}
$$

This large-scale optimisation problem is nonconvex due to the nonlinear activation dynamics and therefore features local optima. Hereafter it is shown that by a nonlinear change of variables, the nonlinear constraints (5) or (6) can be replaced by linear constraints, such that the nonconvex optimisation problem turns into a convex quadratic optimisation problem with a single optimal value.

A nonlinear change of variables allows expressing the activation dynamics as a set of linear equality and inequality constraints. First, the new optimisation variables:

$$
v_{j}=\frac{\mathrm{d} a_{j}}{\mathrm{~d} t}
$$

are introduced, along with the additional constraints:

$$
v_{j}\left(t_{k}\right)=\frac{a_{j}\left(t_{k+1}\right)-a_{j}\left(t_{k}\right)}{t_{k+1}-t_{k}} .
$$


These constraints enforce that $v_{j}$ is the time derivative of the muscle activation $a_{j}$. Second, expressing both (3) and (5), and (3) and (6) in terms of $v_{j}$ gives rise to:

$$
\begin{gathered}
v_{j}+\frac{a_{j}}{\tau_{d}} \geq 0 ; \\
v_{j}+\frac{a_{j}}{\tau_{a}} \leq \frac{1}{\tau_{a}} .
\end{gathered}
$$

By introducing $v_{j}$ and the constraints (13), (14), and (15), the variable $u_{j}$ and constraints (3) and (5) or (6) are eliminated from the optimisation problem. The optimisation problem is now a convex quadratic optimisation problem (QP): the new set of optimisation variables is $v_{j}, a_{j}$, and $F_{M T, j}$; the objective function (11) is quadratic; the inequality constraints $(4,14,15)$ and the equality constraints $(9$, 13, and the constraints enforcing the MT-forces to be consistent with the joint reaction moments) are linear.

After solving the optimisation problem, $u_{j}$ is calculated from $v_{j}$ and $a_{j}$ using either (5) or (6):

Zajac:

$$
u_{j}=\frac{v_{j}+\frac{a_{j}}{\tau_{d}}}{\frac{1}{\tau_{a}}+\left(\frac{1}{\tau_{d}}-\frac{1}{\tau_{a}}\right) a_{j}} ;
$$

Raasch:

$$
u_{j}=\left\{\begin{array}{ll}
\tau_{d} v_{j}+a_{j} & v_{j} \leq 0 \\
\frac{a_{j} c_{1}-c_{2}+\sqrt{D}}{2 c_{1}} & v_{j}>0
\end{array},\right.
$$

where $c_{1}=\frac{1}{\tau_{a}}-\frac{1}{\tau_{d}}, c_{2}=\frac{1}{\tau_{d}}$, and $D=\left(c_{2}+a_{j} c_{1}\right)^{2}+4 c_{1} v_{j}$. Figure 3 visualizes this change of variables. Muscle excitations based on the activation dynamics model of Raasch et al. (1997) (17) are further reported.

\subsection{Post-Processing of the CIA}

The muscle excitations corresponding to the activations predicted by the CIA, are calculated by (17). Since the CIA does not enforce the muscle activations to comply with muscle physiology, this computation is not guaranteed to yield a physiologically meaningful excitation, that is, a real value between 0 and 1 .

\subsection{Validation}

The calculated excitations are compared to the experimentally collected, high-pass filtered and rectified surface EMG data $(1000 \mathrm{~Hz})$ of 8 muscle groups.

\section{Results}

\subsection{Computational results}

All optimisation problems are solved on a Pentium M, $2 \mathrm{GHz}$ processor with $1 \mathrm{~GB}$ RAM using the KNITRO Interior/Direct algorithm (Byrd et al. 2006). KNITRO 

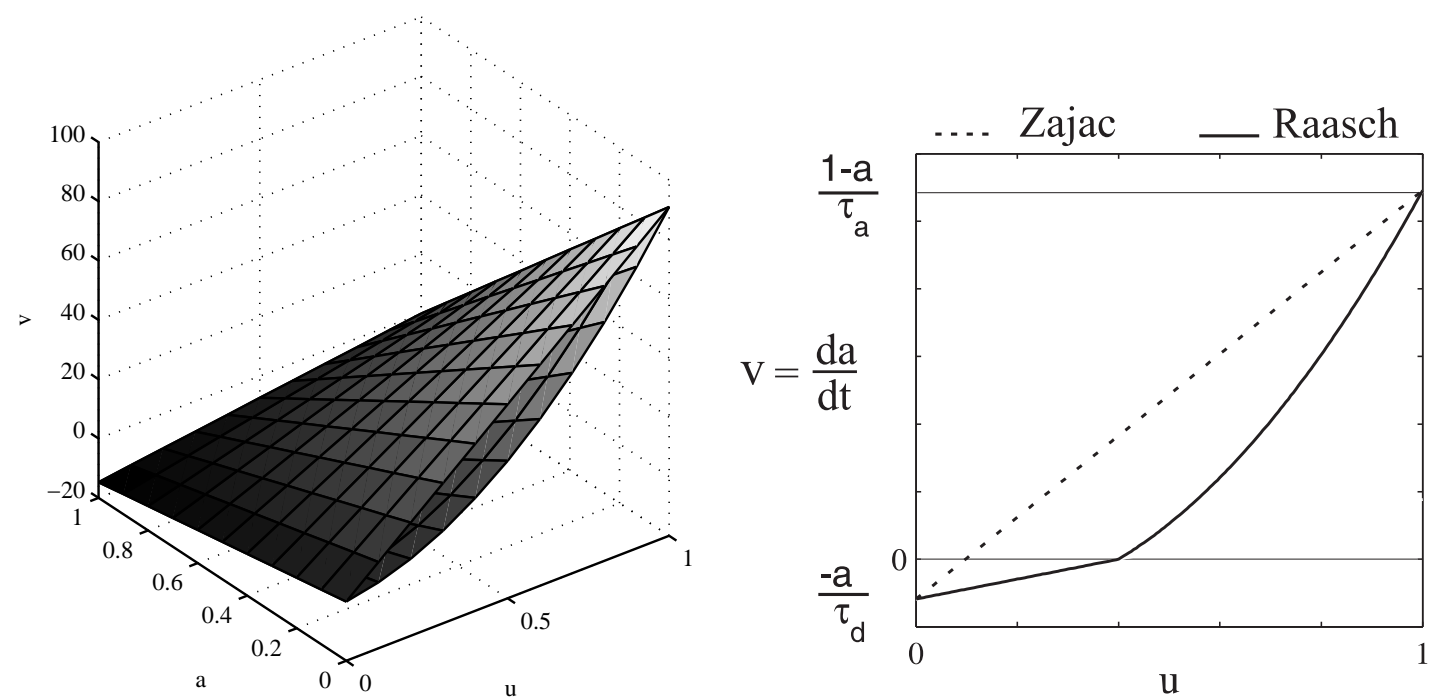

Figure 3. Nonlinear change of variables. Relation between the excitation $u$ and the time derivative of the activation $v$ for $0 \leq a \leq 1$ (left) and $a=0.4$ (right) as defined by the activation dynamics according to Zajac (1989) (top, dashed line) and Raasch et al. (1997) (bottom, solid line). Both models give rise to a different description of the activation dynamics: the surfaces (left) and the solid and dashed line (right) do not coincide. However, the time derivative of activation is subject to the same limits: the surfaces intersect for $u=0$ and $u=1$ (left) and the minimum and maximum value of $v$ are equal for the solid and dashed line (right). The intersections of the surfaces (left) are straight lines: the change in activation is linearly constrained.

Table 1. Computational data of the optimisation problems underlying the CIA and the PIA
for the control (C) and hemiparetic $(\mathrm{H})$ subjects. Whereas the PIA requires solving one large
scale quadratic program (QP), the CIA requires solving multiple small QPs, one for each time
instant $t_{k}$. For each subject and method, the number of QPs, the number of variables per QP,
the number of function evaluations (NFE) averaged over all QPs, and the summed CPU time in
seconds of all QPs are reported.
$\begin{aligned} & \text { Subject } \\ & \text { Sethod }\end{aligned}$

offers the choice between interior-point and active-set methods. The interior-point method is faster than the active-set method on the optimisation problem underlying the PIA. This is in accordance with the statement reported in (Nocedal and Wright 2006) that interior-point methods are generally much faster on large convex quadratic problems than active-set methods. Table 1 summarizes the computational data of the optimisation problems underlying the CIA and the PIA for the control and hemiparetic subjects.

\subsection{Comparison of the excitations computed by the PIA and the experimental EMG}

Figure 4A-B and Figure 5A-B show the kinematics and kinetics used as input data for the simulations. Figure 4C and Figure 5C compare the average linear enveloped EMG to the calculated muscle excitations. A good agreement in timing is observed for the major muscle groups during normal and hemiparetic gait.

\subsubsection{Control subject}

Excitation of M. soleus and M. gastrocnemius during mid stance and pre swing is predicted in agreement with the measured EMG. The onset of M. soleus preceeding 
the onset of M. gastrocnemius is accounted for.

M. tibialis anterior presents maximal excitation during stance-swing transition, with continued excitation during swing. The duration of the predicted excitation during loading response is however shortened. The increase in excitation prior to initial contact is consistent with the surface EMG.

During loading response, maximal excitation of $\mathrm{M}$. vastus lateralis is confirmed. For M. rectus femoris, delayed onset of excitation during loading response is present as well as a limited level of excitation preceeding the stance-swing transition. During terminal swing, the increased excitation of these muscles observed in the surface EMG is not predicted.

Although high M. gluteus medius excitation is calculated during the entire stance phase, experimental EMG only indicates excitation in the early part of stance.

The computed excitations of M. biceps femoris and M. semimembranosus relate closely to the measured EMG patterns during initial contact and terminal swing. However, for both muscles the predicted excitations around stance-swing transition are not confirmed in the surface EMG.

\subsubsection{Hemiparetic Subject}

The timing of peak excitation of M. gastrocnemius and M. soleus is predicted correctly by the PIA. The duration of the computed excitations is however short compared to the experimental EMG. The delayed onset of the M. soleus excitation, as present in the surface EMG, is reproduced correctly.

The prolonged excitation of M. tibialis anterior through mid stance, as observed in the EMG recordings, is confirmed by the calculated muscle excitations. An additional excitation burst at the stance-swing transition is calculated, but the timing of peak excitation is premature compared to the experimental EMG.

The nearly continuous excitation of the M. rectus femoris calculated in stance is confirmed by the recorded EMG signals. The additional burst in swing is only minimally present in the calculated excitations.

The continuous low level excitation of M. vastus lateralis present in the experimental data during stance is not confirmed.

The PIA correctly predicts the experimentally observed excitation burst of M. semimembranosus and M. biceps femoris during stance-swing transition. The excitation burst of both muscles measured at terminal swing and initial contact is not present in the calculated excitations.

Although the computed excitation and the recorded surface EMG of the M. gluteus medius show good agreement during first half of stance, the calculated muscle excitation tends to show a delay as well as a second excitation burst during second half of stance.

\subsection{Comparison of the calculated MT-forces using the CIA and PIA}

Figure 6 and Figure 7 present the excitations and corresponding MT-forces computed by the PIA and the CIA for a selection of muscles. The vertical grey bands indicate the time instants where too steep an increase or decrease in MT-force is predicted in the CIA and therefore physiologically meaningful excitations do not exist. This only occurs at a limited number of time instants, mainly related to stance-swing transition.

For all muscles, the MT-force decay rate predicted by the PIA is significantly slower than predicted by the CIA. The PIA consistently predicts earlier cessation in the excitations, as most clearly observed in M. tibialis anterior (terminal stance) and M. soleus (pre swing) of the hemiparetic subject. Furthermore, the slower 
A) Kinematics
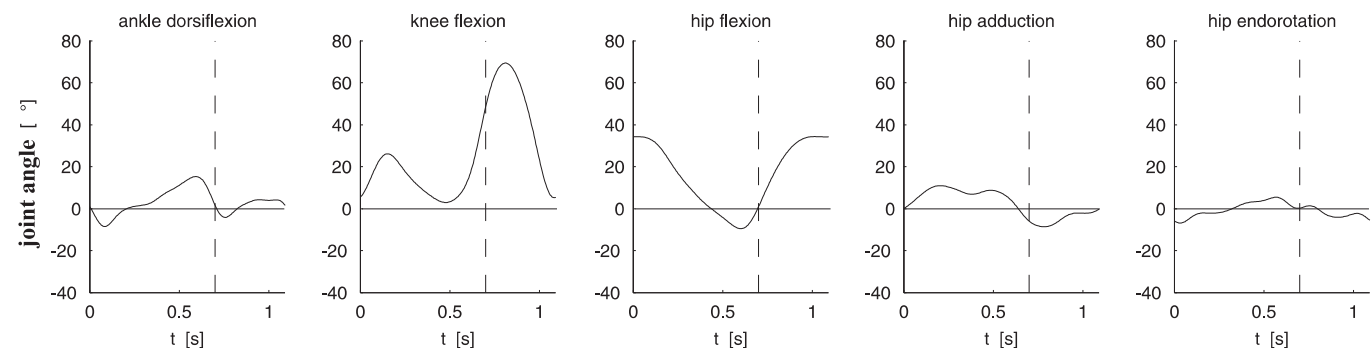

B) Kinetics (internal moments)
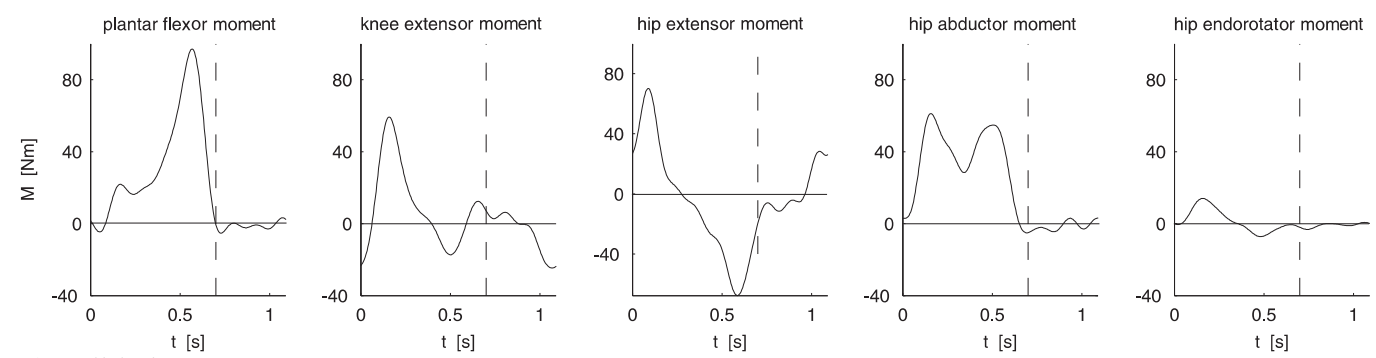

C) Validation
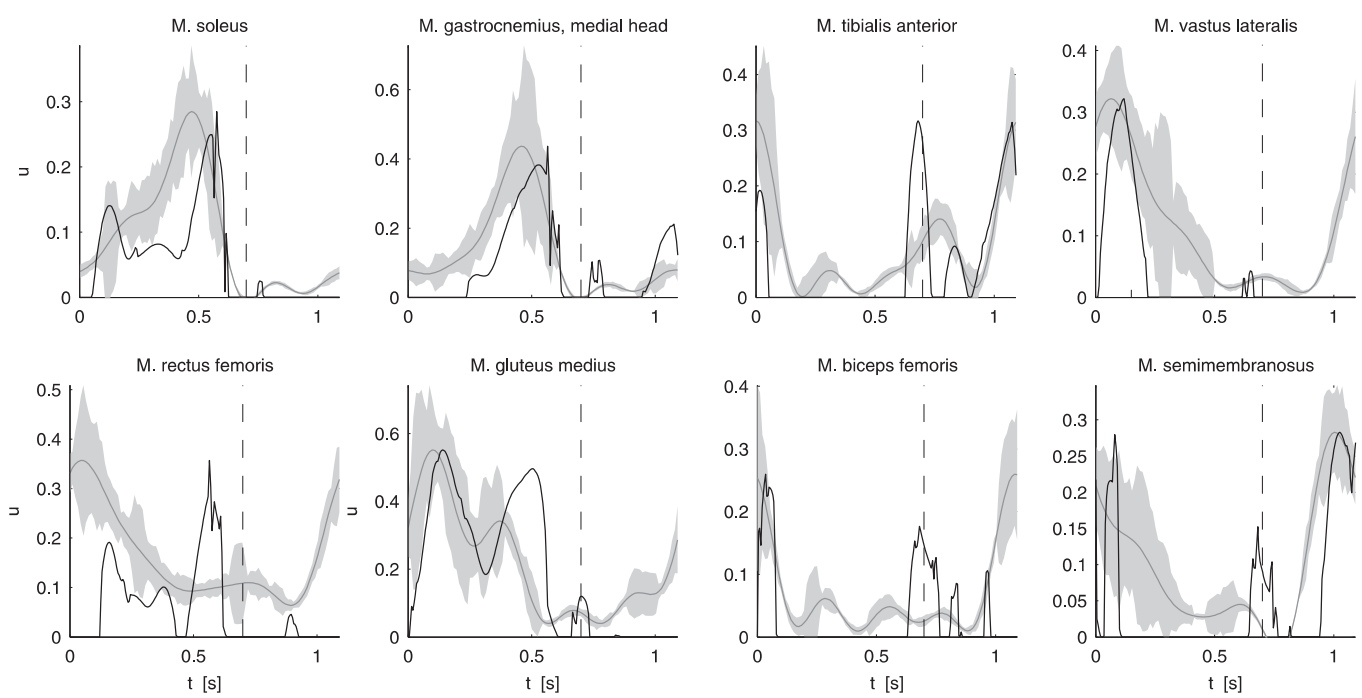

Figure 4. Control simulation: input data and results of the physiological inverse approach. Figure A and $\mathrm{B}$ show, respectively, the kinematics and kinetics of ankle, knee, and hip of the control subject, used as input data for the simulation. Figure $\mathrm{C}$ compares the muscle excitations computed by the physiological inverse approach (black line) with the experimental EMG (grey line). The averaged EMG is scaled to the maximum excitation level during the gait cycle. The standard deviation of the EMG is indicated by the grey band.

force decay in M. gastrocnemius results in a higher and earlier onset of M. tibialis anterior excitation, increasing the duration of co-contraction between plantarflexors and dorsiflexors.

The PIA predicts passive force generation in M. soleus and M. gastrocnemius during second half of stance and in M. rectus femoris around stance-swing transition. To account for the lack of passive force production in the CIA, higher levels of muscle excitation are predicted for M. soleus and M. gastrocnemius to achieve similar force production. The findings for M. rectus femoris are different. The excitations predicted by the CIA for M. rectus femoris are not significantly higher than those predicted by the PIA. Instead, the PIA introduces additional excitations in 
A) Kinematics
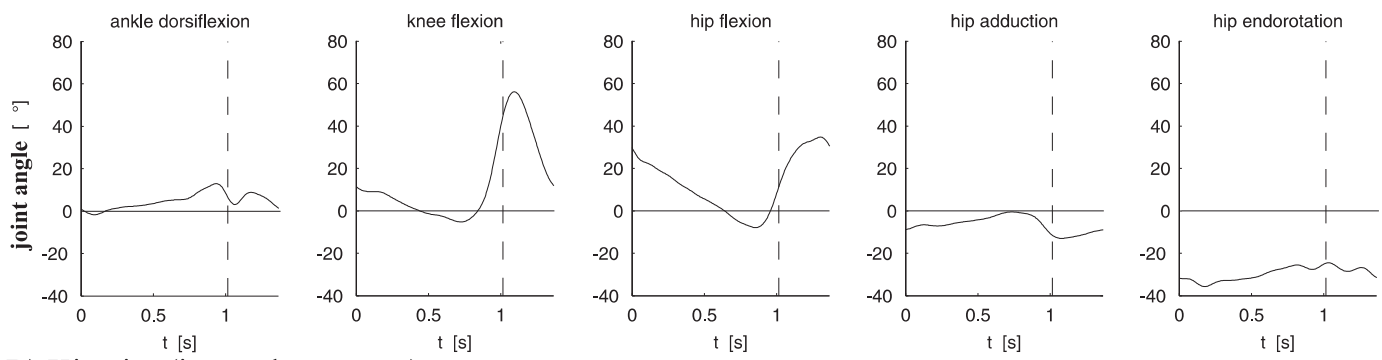

B) Kinetics (internal moments)
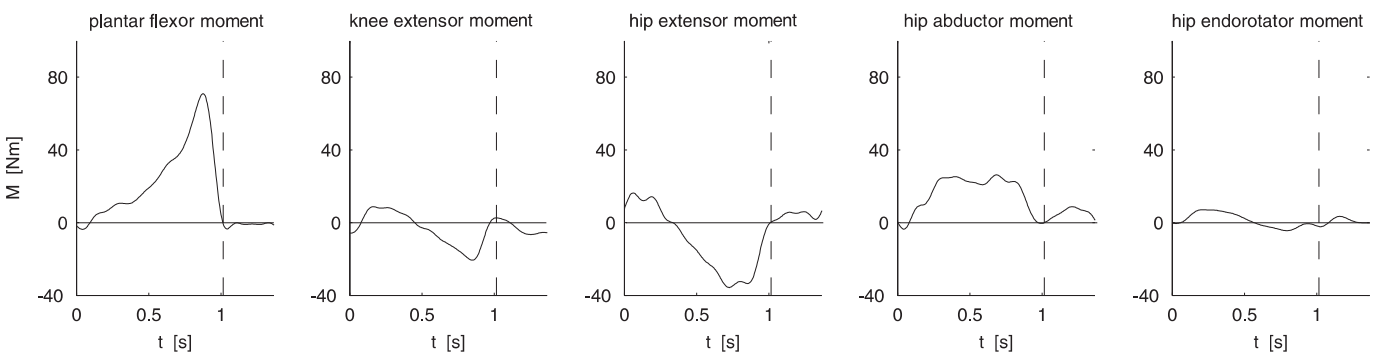

C) Validation
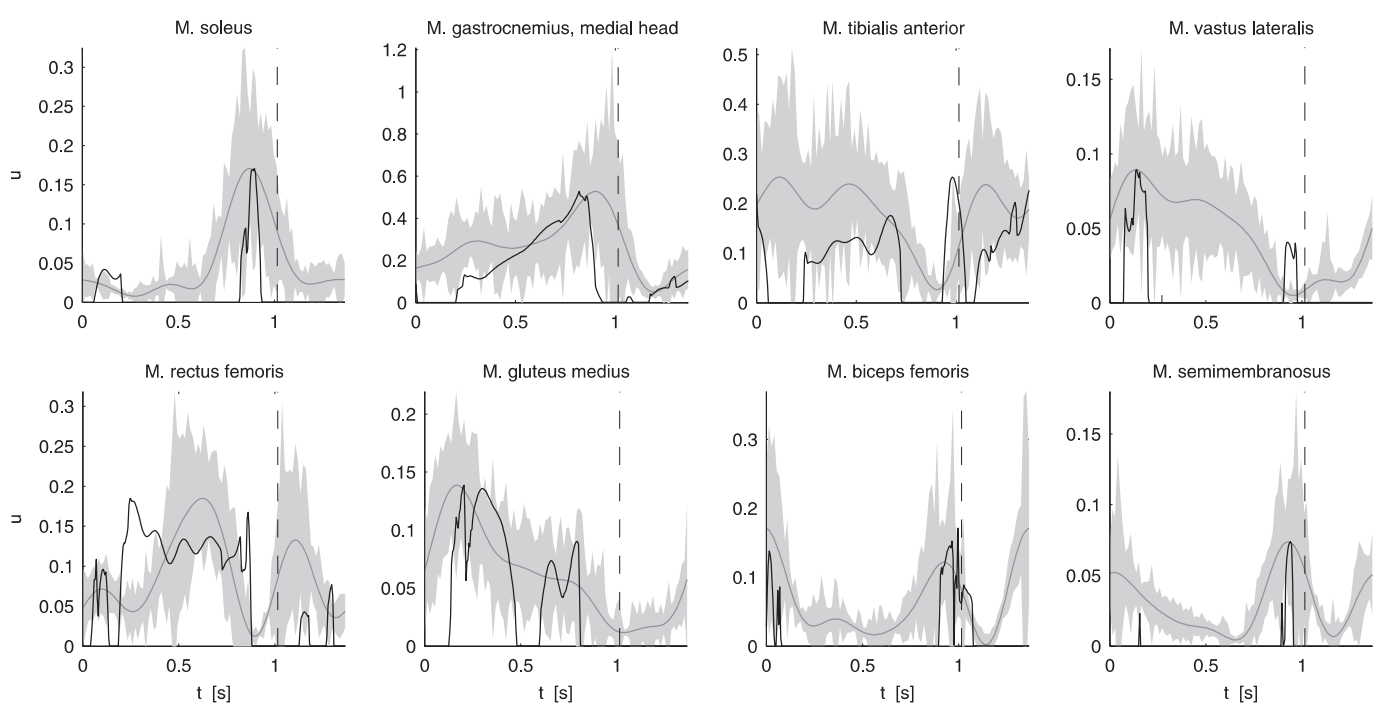

Figure 5. Hemiparetic simulation: input data and results of the physiological inverse approach. Figure $\mathrm{A}$ and $\mathrm{B}$ show, respectively, the kinematics and kinetics of ankle, knee, and hip of the hemiparetic subject, used as input data for the simulation. Figure $\mathrm{C}$ compares the muscle excitations computed by the physiological inverse approach (black line) with the experimental EMG (grey line). The averaged EMG is scaled to the maximum excitation level during the gait cycle. The standard deviation of the EMG is indicated by the grey band.

the antagonistic muscle group, M. biceps femoris, to balance the passive force production of M. rectus femoris at knee level. These findings are more pronounced in the control data than in the hemiparetic data.

\section{Discussion}

This paper investigates the potential of the physiological inverse approach (PIA) to calculate MT-forces underlying an experimentally measured movement pattern, here gait. This approach supplements the classical inverse approach (CIA) with 

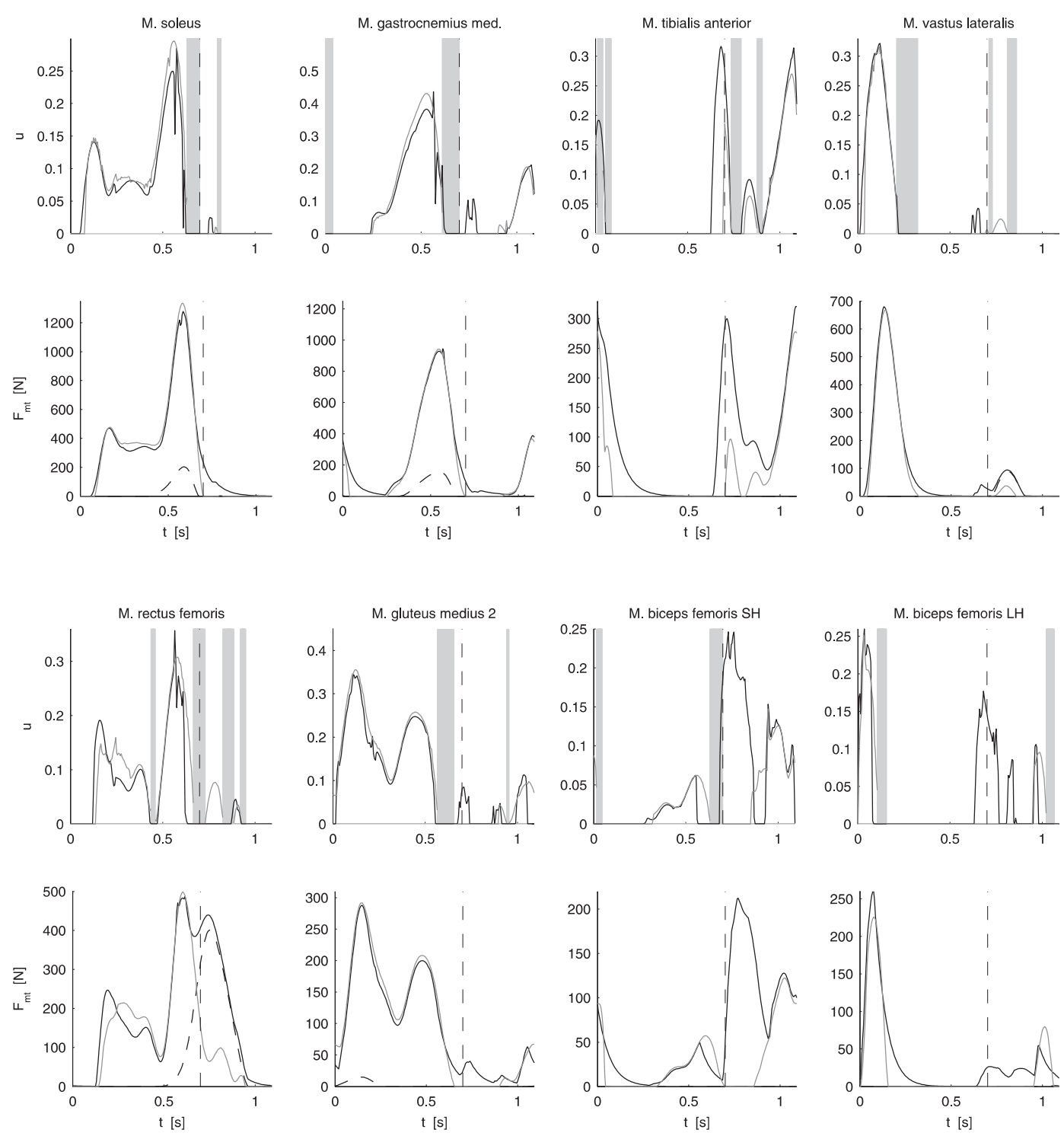

Figure 6. Control simulation: comparison of the classical and the physiological inverse approach. Comparison of the muscle excitations and MT-forces of the classical (grey line) and physiological inverse approach (black line) for the control subject. The muscle excitations are shown above the corresponding MT-forces. The passive force component of the PIA-results is indicated by a dashed line. The time instants for which no physiological meaningful excitation, that is, a value of $u$ between 0 and 1 , can be computed for the CIA-results are indicated in grey.

constraints imposed by muscle physiology. Using convex optimisation techniques, the underlying nonlinear optimisation problem is reformulated as an equivalent convex optimisation problem which can be solved efficiently.

The proposed nonlinear change of variables which linearizes the activation dynamics does not change the global optimum of the optimisation problem, since the one-to-one relation between muscle excitation $u_{j}$ and the variable $v_{j}$ guarantees that for every solution in $u_{j}$ a corresponding unique solution in $v_{j}$ exists, and vice versa. However, by performing the change of variables a convex QP instead of a nonconvex program is obtained. The major advantage is that a convex QP has a single optimum instead of many local optima in which solvers get trapped. Furthermore, there exist very efficient algorithms to solve QPs.

By introducing the nonlinear change of variables, we additionally show that the activation dynamics models proposed by Zajac (1989) and Raasch et al. (1997) are 



Figure 7. Hemiparetic simulation: comparison of the classical and the physiological inverse approach. Comparison of the muscle excitations and MT-forces of the classical (grey line) and physiological inverse approach (black line) for the control subject. The muscle excitations are shown above the corresponding MT-forces. The passive force component of the PIA-results is indicated by a dashed line. The time instants for which no physiological meaningful excitation, that is, a value of $u$ between 0 and 1 , can be computed for the CIA-results are indicated in grey.

equivalent in terms of muscle activations and MT-forces. Although both models give a different description of the activation dynamics (solid and dashed line in Figure 3 do not coincide), the rate of change in activation is subject to the same limits (minimum and maximum value of $v$ are equal for the solid and dashed line in Figure 3). At the level of the optimisation problem this observation is reflected in (3) and (5) giving rise to the same constraints $(14,15)$ as (3) and (6). In an inverse approach, there are no additional constraints on the excitations and as a consequence, both models give rise to the same values for the muscle activations and the MT-forces at the global optimum; however, the corresponding muscle excitations are different, (16) versus (17).

In general, the muscle excitations computed by the PIA show good agreement with key features of muscle actions during normal gait (Gage 1991; Perry 1992). The role of M. gastrocnemius and M. soleus during stance, the antagonistic action of M. tibialis anterior during initial contact and swing, as well as the excitation 
of the hamstrings around terminal swing and initial contact are confirmed. The excitation burst for M. gluteus medius is longer than experimentally observed, which might be attributed to insufficient selectivity in electrode placement on this large muscle group.

For hemiparetic gait, key pathological timing abnormalities present in the recorded EMG are predicted: prolonged excitation of $\mathrm{M}$. tibialis anterior and M. rectus femoris during mid stance, as well as the excitation burst of M. semimembranosus and M. biceps femoris around stance-swing transition. The low activation of M. vastus lateralis during stance is not confirmed, since it is mainly M. rectus femoris that accounts for the knee extension moment. The additional excitation burst of M. rectus femoris during swing phase is not as pronounced as experimentally observed. While similarities between excitations and EMG are obtained in the hemiparetic subject, based on input kinematics and kinetics and the assumption of optimal load sharing, the remaining differences between excitations and EMG mentioned above, indicate that the assumption of optimal load sharing is inadequate in these patients. Hence, accurate calculation of muscle excitations during hemiparetic gait requires the modelling and incorporation of neural control.

This paper proposes a post-processing step for the CIA which computes the corresponding muscle excitations. However, even for slow motions like gait this post-processing cannot yield muscle excitations at all time instants, since the CIA does not enforce the computed MT-force patterns to comply with muscle physiology. While these excitation results are therefore inherently partial, they are judged valuable. First, a more accurate assessment of the predicted timing of muscle action is possible through direct comparison of the muscle excitations (instead of the calculated MT-forces) with the measured EMG. Second, comparing the PIA and CIA excitation patterns allows a more profound investigation of the effect of muscle physiology.

Comparison of the CIA and PIA results reveals that during gait both activation and contraction dynamics affect the muscle excitations and MT-force patterns, hereby altering the level of co-contraction predicted by the simulation.

The activation dynamics limits the rate of muscle force build-up and, especially, decay. The fast decrease in MT-force of M. gastrocnemius and M. soleus predicted by the CIA is inconsistent with muscle physiology. Enforcing a gradual force buildup and decay in the PIA influences the onset and cessation of muscle excitations, as most clearly observed in the earlier excitation cessation of M. tibialis anterior and M. soleus of the hemiparetic subject. Furthermore, the predicted degree of cocontraction between antagonistic muscle groups changes, especially during phases that precede and follow loading response and pre swing, in which ground reaction forces change rapidly (Thelen and Anderson 2006; Neptune and Kautz 2001). For instance, a higher and premature excitation of M. tibialis anterior is required to achieve sufficient force production associated with ankle control during swing in the presence of the decaying muscle force of the ankle plantarflexors.

By including the contraction dynamics in the PIA, the passive component of the muscle force is accounted for. The plantarflexors produce passive force during the second half of stance, while M. rectus femoris yields substantial passive force during initial swing. The force output without excitation gives rise to substantial co-contraction of antagonistic muscle groups, e.g., M. biceps femoris versus M. rectus femoris. In contrast, the CIA requires additional excitation bursts to achieve sufficient MT force production. The high level of passive muscle force for M. rectus femoris $(\mathrm{C})$ is remarkable and suggests that customization of the generic muscle parameters, which affect the ratio between active and passive muscle force, may become more crucial when using the PIA. 
Although limited, we value the observed effects of muscle physiology on the muscle excitations and MT-force patterns as relevant. This conclusion is in marked contrast with Anderson and Pandy (2001) who judge static and dynamic optimisation solutions for gait to be practically equivalent. The static solution of Anderson and Pandy (2001) is based on the same CIA implementation as used here, while the dynamic solution, which includes muscle physiology, is based on a forward dynamic simulation. The discrepancy in conclusion relates to the additional computational effort required for predicting MT-forces consistent with muscle physiology. Our approach gives rise to a limited additional effort, since the PIA requires only marginally more computational time than the CIA. Anderson and Pandy (2001), on the other hand, need to carry out a forward dynamic simulation of which the additional computational effort is huge compared to the CIA, and therefore not justifiable in view of the limited effect on the computed MT-force patterns.

Given its dynamic nature, the PIA allows incorporation of physiological cost functions such as muscle fatigue and metabolic energy usage, of which the specific effect on the predicted excitations and MT-force patterns is to be explored.

The observations presented here relate only to gait. In explosive movements such as jumping, cycling and sprinting, the impact of muscle physiology is more prominent and the contribution of active versus passive force production may be altered. Future work includes assessing the potential of the PIA for such movements. This will require, among other things, the investigation of the effect of muscle fiber contraction speed on the MT-force, neglected in this paper.

By incorporating the effect of muscle contraction speed, the contraction dynamics are described by a first order dynamic model instead of the linear approximation for slow motions (9). Extending the nonlinear change of variables to the contraction dynamics results in a set of inequality constraints limiting the first and second time derivatives of the musculotendon forces. However, these inequality constraints are no longer convex in the optimisation variables and consequently, no computational advantage is obtained.

In conclusion, the PIA supplements aspects of muscle activation and contraction dynamics to the CIA without substantial increase of the computational time. By a nonlinear change of variables the activation dynamics can be efficiently handled in the PIA. The MT-forces are the solution of a QP of which the global optimum is found with high computational efficiency. Simulations for normal and pathological gait show that our approach constitutes a promising improvement of the CIA. Inclusion of muscle physiology mainly affects the rate of muscle force build-up and decay, as well as the level of passive force production, both influencing the level of co-contraction.

\section{Acknowledgements}

F. De Groote and G. Pipeleers are Research Assistants of the Research Foundation - Flanders (FWO-Vlaanderen). I. Jonkers and B. Demeuelenaere are Postdoctoral Fellows of the Research Foundation - Flanders. I. Jonkers receives additional funding from the Belgian Educational Foundation and the Koning Boudewijn Fonds. The support of the following projects is gratefully acknowledged: project G.0462.05 of the Research Foundation - Flanders, K.U.Leuvens Interdisciplinary Research Program IDO/07/12, and K.U.Leuven-BOF EF/05/006 Center-of-Excellence Optimization in Engineering. The support of VA RR\&D Merit Review Grant No. B2792RT is acknowledged. The scientific responsibility remains with its authors. 


\section{References}

Anderson FC. 1999. A dynamic optimization solution for a complete cycle of normal gait[Ph.D. dissertation]. University of Texas. Bell and Howell Information and Learning Company.

Anderson F, Pandy M. 2001. Static and dynamic optimization solutions for gait are practically equivalent. Journal of Biomechanics 34:153-161.

. 2001. Dynamic optimization of human walking. Journal of Biomechanical Engineering 123(5):381390.

Boyd S, Vandenberghe L, editors. 2004. Convex Optimization. Cambridge University Press.

Byrd RH, Nocedal J, Waltz RA. 2006. "KNITRO: An Integrated Package for Nonlinear Optimization," Large-Scale Nonlinear Optimization. Springer-Verlag. pp. 35-59.

Crowninshield R, Brand R. 1981. A physiologically based criterion of muscle force prediction in locomotion. Journal of Biomechanics 14(11):793-801.

De Groote F, Pipeleers G, Jonkers I, Demeulenaere B, Swevers J, De Schutter J. 2006. A physiology based inverse dynamic analysis of musculotendon forces during human movement.In: Journal of Biomechanics. Munich, Germany. July - August 2006. 39(S1). p. S45.

de Leva P. 1996. Adjustments to Zatsiorsky-Seluyanovs segment inertia parameters. Journal of Biomechanics 29(9):1223-1230.

Delp S, Loan J, Hoy M, Zajac F, Topp E, Rosen J. 1990. An interactive graphics-based model of the lower extremity to study orthopaedic surgical procedures. IEEE Transactions on Biomedical Engineering 37(8):757-767.

Gage J, editor. 1991. Gait analysis in cerebral palsy. London: Mac Keith Press.

Hatze H. 1976. The complete optimization of human motion. Mathematical Biosciences 28:99-135.

. 1981. A comprehensive model for human motion simulation and its application to the take-off phase of the long jump. Journal of Biomechanics 14(3):135-142.

Lieber R, editor. 1992. Skeletal Muscle Structure and Function: Implications for Rehabilitation and Sports Medicine. Willians and Wilkins.

Neptune R, Kautz S. 2001. Muscle activation and deactivation dynamics: the governing properties in fast cyclical human movement performance?. Exercise and Sport Science Reviews 29(2):76-80.

Nocedal J, Wright SJ. 2006. "Quadratic Programming," Numerical Optimization. Second ed. Springer. p. 490 .

Perry J, editor. 1992. Gait analysis: Normal and pathological function. SLACK incorporated.

Pipeleers G, Demeulenaere B, Jonkers I, Spaepen P, Van der Perre G, Spaepen A, Swevers J, De Schutter J. 2008. Dynamic simulation of human motion: numerically efficient inclusion of muscle physiology by convex optimization. Optimization and Engineering 9(3):213-238.

Raasch C, Zajac F, Ma B, Levine W. 1997. Muscle coordination of maximum-speed pedaling. Journal of Biomechanics 30(6):595-602.

Sutherland D. 2002. The evolution of clinical gait analysis Part II: Kinematics. Gait and Posture 16:159179.

Thelen D, Anderson F. 2006. Using computed muscle control to generate forward dynamic simulations of human walking from experimental data. Journal of Biomechanics 39(6):1107-1115.

Winters J, Stark L. 1988. Estimated mechanical properties of synergistic muscle involved in movements of a variety of human joints. Journal of Biomechanics 21(12):1027-1041.

Yamaguchi G, Moran D, Si J. 1995. A computational efficient method for solving the redundant problem in biomechanics. Journal of Biomechanics 28(8):999-1005.

Yamaguchi G, Zajac F. 1989. A planar model of the knee joint to characterize the knee extensor mechanism. Journal of Biomechanics 22(1):1-10.

. 1990. Restoring unassisted natural gait to paraplegics via functional neuromuscular stimulation: a computer simulation study.. IEEE Transactions on Biomedical Engineering 37(9):886-902.

Zajac FE. 1989. Muscle and tendon: properties, models, scaling and application to biomechanics and motor control. Critical Reviews in Biomedical Engineering 17(4):359-411. 